\title{
Maternal prolactin concentrations and lactational behaviour in the early postpartum period in women with lactational amenorrhoea
}

\author{
Kamani H. Tennekoon ${ }^{1}$ \\ (Index words: Lactational amenorrhoea, prolactin, lactational behaviour).
}

\begin{abstract}
Objective To compare early postpartum differences in maternal prolactin concentrations and lactational behaviour between women who have short and long periods of lactational amenorrhoea.
\end{abstract}

Methods Healthy lactating women resuming regular menstruation before 24 weeks [short amenorrhoea (SA) group; $\mathrm{n}=15$ ], and at or after 24 weeks [long amenorrhoea (LA) group; $n=15$ ] postpartum were studied from 4 to 12 weeks postpartum. Infant feeding pattern and maternal plasma prolactin concentrations were compared between the two groups using repeated measures ANOVA.

Results Breastfeeds/24 h were significantly higher and other feeds/ $24 \mathrm{~h}$ were significantly lower $(\mathrm{p}<0.01)$ at 12 weeks postpartum in the LA group when expressed as a percentage of all feeds. Number of breastfeeds (total/24 h and night) significantly decreased and the number of other feeds significantly increased ( $p<0.05$ to $0<0.001)$ with time in both groups. Prolactin concentrations (basal, suckling stimulated and increment) were higher in the LA group, but consistent significant differences were observed mainly in the increment $(p<0.05$ to $p<0.001)$. Prolactin concentrations significantly $(p<0.01$ to $<0.001$ ) decreased with time postpartum in the SA group, but not in the LA group.

Conclusions Women with longer periods of lactational amenorrhoea maintain higher prolactin concentrations than those with shorter periods of lactational amenorrhoea during the early postpartum period, even when the breastfeeding frequency is reduced. This could be explained by a greater sensitivity of the pituitary lactotrophe to the suckling stimulus in women who have longer periods of lactational amenorrhoea.

\section{Introduction}

Lactational amenorrhoea, which contributes significantly to curtailing population growth, is known to be affected by many factors. Some of these are frequency and duration of breastfeeding $(1,2,3)$, introduction of supplementary feeds to the infant (4), maternal nutritional status $(5,6,7,8)$ and previous duration of lactational amenorrhoea (9).

A World Health Organization multinational study has shown that, in addition to cross-cultural effects, infant feeding characteristics and non-feeding variables such as parity, maternal body mass index (BMI) and frequency of infant illness also affect the duration of lactational amenorrhoea $(10,11)$. However, there are reports of variations in the duration of lactational amenorrhoea even among women with similar nursing patterns (12). It is possible that women respond variably to the stimulus of suckling, leading to endocrine differences affecting the activity of the hypothalamic-pituitary-ovarian axis, that may contribute to variations in the duration of lactational amenorrhoea. High plasma prolactin and low plasma oestrogen concentrations have been observed in women with longer periods of lactational amenorrhoea in some studies (13), whereas others (14) have failed to observe any association between plasma prolactin and duration of lactational amenorrhoea. The present study was done to investigate early postpartum variations in plasma prolactin concentrations and infant feeding patterns between women who resume regular menstruation early and late in the postpartum period.

\section{Materials and methods}

Thirty healthy lactating women were studied after obtaining their informed consent and institutional ethical approval. They were between 20 and 35 years of age, in para 2 or 3 , and had BMIs ranging from 18 to $27 \mathrm{~kg} / \mathrm{m}^{2}$. Characteristics of the sample have been described elsewhere, as the women used for this analysis were the controls in a study on maternal nutritional supplementation and lactational amenorrhoea (15). Each of them, had delivered a single, healthy, term baby by spontaneous or assisted vaginal delivery at the end of an uncomplicated pregnancy. They were non-working mothers, exclusively breastfeeding at the time of recruitment, had breast fed their previous baby/babies, were not using any form of hormonal contraceptives and were living within a $20 \mathrm{~km}$ radius from the institution. They were recruited from the postpartum wards of the De Soysa Hospital for Women within $24 \mathrm{~h}$ of delivery. The study commenced at 4 weeks postpartum. Those who had introduced other feeds to the infants or planned to use hormonal contraceptives by this time were excluded.

They were studied from 4 weeks postpartum till they had 2 to 3 regular menstrual bleeds. If the interval between two consecutive menstrual bleeds was more than 3 weeks and less than 6 weeks, the first of these was taken as a regular menstrual bleed. Those who resumed regular men-

'Associate Professor in Physiology, Faculty of Medicine, University of Colombo. (Accepted 2 September 2000. This work was supported by grant No: 88008 of the Task Force on Natural Methods for Fertility Regulation, Special Programme on Research, Development and Research Training in Human Reproduction, World Health Organization). 
struation before 24 weeks postpartum were considered as having short periods of lactational amenorrhoea (SA group), and those who resumed menstruation at or after 24 weeks postpartum as having long periods of lactational amenorthoea (LA group). There were 15 subjects in each group.

Although the subjects were divided according to the period of lactational amenorrhoea retrospectively, data were collected prospectively at 4,8 and 12 weeks postpartum. Information on infant feeding patterns and menstrual status was obtained during home visits made at weekly intervals. Breastfeeds given from 06.00 to $22.00 \mathrm{~h}$ (both hours inclusive) were taken as daytime feeds while the remainder was considered as night feeds. Formula feeds and caloric liquids were considered as other feeds. Feeds of fruit juice and other non-caloric liquids comprised less than $15 \%$ of all feeds and were not considered as other feeds.

Blood samples for prolactin estimation were collected at 4-weekly intervals. The sample for basal prolactin estimation was collected between 14.00 and $15.00 \mathrm{~h}$, at least 120 min after the previous breastfeed. The suckling stimulated sample was collected $\mathbf{3 0} \mathrm{min}$ after the commencement of a breastfeed given after collecting the basal sample. Plasma prolactin concentrations were measured by double antibody radioimmunoassay using World Health Organisation matched reagents (16). The average batch coefficient variation was $6.6 \%$, and the inter-assay variations were $10.3 \%, 7.3$ and $9.0 \%$ for low, medium and high quality control pools respectively. All samples from each subject were analysed within one assay.

The number of breastfeeds (total $/ 24 \mathrm{~h}$, daytime, night and total expressed as a percentage of all feeds), number of other feeds (total/24 $\mathrm{h}$ and expressed as a percentage of all feeds), prolactin concentrations [basal, suckling stimulated and the increment of prolactin (ie. suckling stimulated - basal)], maternal BMI and infant weights (expressed as a percentage of birth weight) from 4 to 12 weeks postpartum were compared between the two groups using repeated measures ANOVA. Bonferroni multiple comparison test for selected pairs was used to compare the two groups at 4,8 and 12 weeks postpartum while post-test for linear trend was used to look at the effect of time postpartum within each group. Birth weights of the infants were compared using the Students $t$ test. Previous history of short lactational amenorrhoea, parity and the infant sex between the two groups were compared using the Chi square test with Yates correction for small numbers.

\section{Results}

The baseline characteristics of the two groups are shown in Table 1. Maternal age and BMI did not differ significantly between the two groups although the mean maternal BMI was lower in the LA group. There was a higher number of women with a previous history of short lactational amenorrhoea (ie. $<24$ weeks) and women in para 3 in the SA group. Number of male infants was higher in the LA group. Infant weight was higher in the SA group. These differences were not statistically significant.

Total number of breastfeeds $/ 24 \mathrm{~h}$, number of daytime breastfeeds, night breastfeeds and other feeds $/ 24 \mathrm{~h}$ in both groups by time postpartum (mean \pm SEM) are shown in Table 2 . The total number of breastfeeds/24 h was not significantly different between the two groups. However, when these were expressed as a percentage of all feeds $/ 24 \mathrm{~h}$, the percentage of feeds constituted by breast milk was significantly different between the two groups $(p<0.0001)$. This difference was attributed a significantly higher percentage ( $p<0.01$ ) of breastfeeds in the LA group (mean \pm SEM: $96.11 \pm 1.56$ ) than in the SA group (mean \pm SEM: 88.83 \pm 3.22 ) at 12 weeks postpartum. Number of daytime and night breastfeeds was not significantly different between the two groups.

Table 1. Baseline characteristics of women who resumed menstruation before 24 weeks (SA group) and at or after 24 weeks (LA group) postpartum

\section{Characteristic}

Maternal age in year (mean \pm SEM)

Duration of previous

lactational amenorrhoea

Parity

Maternal BMI in $\mathrm{kg} / \mathrm{m}^{2}$ (mean \pm SEM)

Infant sex

Birth weights of the infants (g)

(mean+SEM)

$$
\text { SA group }(n=15)
$$

LA group ( $n=15)$

$29.60 \pm 0.74$

$<24$ weeks: $n=13$

$\geq 24$ weeks: $n=2$

Para 2: $n=6$

Para 3: $n=9$

$21.95 \pm 0.84$

Male: $\mathrm{n}=6$

Female: $n=9$

$3007 \pm 167$
$28.00 \pm 1.10$

<24 weeks: $n=8$

$\geq 24$ weeks: $n=7$

Para 2: $n=9$

Para 3: $n=6$

$19.83 \pm 0.65$

Male: $\mathrm{n}=11$

Female: $n=4$

$2808 \pm 127$ 
None of the subjects in either group gave other feeds (excepting fruit juice and non-caloric liquids) to the infants at 4 weeks postpartum. The number of other feeds was significantly different between the two groups $(p<0.0001)$ with the LA group having a significantly lower $(p<0.01)$ frequency at 12 weeks postpartum. This difference remained even when the number of other feeds was expressed as a percentage of all feeds $/ 24 \mathrm{~h}$ (mean \pm SEM: $3.38 \pm 1.54$ in the LA group vs $11.17 \pm 3.22$ in the SA group at 12 weeks postpartum).

Within each group there was a significant decrease in the total number of breastfeeds/24h (SA group $\mathrm{p}<0.001$, LA group $p<0.05$ for linear trend) and in the number of night breastfeeds (SA group, $\mathrm{p}<0.001$; LA group, $\mathrm{p}<0.02$ for linear trend) with time postpartum. However, a similar reduction was not seen for the daytime breastfeeds. The number of other feeds significantly increased with time postpartum in both groups (SA group, $p<0.001$; LA groups, $p<0.05$ for linear trend).

Basal, suckling stimulated and the increment of prolactin concentrations [geometric mean $(95 \%$ confiderice limits)] in the two groups by time postpartum are shown in Table 3. Basal prolactin concentrations were significantly higher $(p<0.0001)$ in the LA group than in the SA group. This difference was attributed to significantly higher val- ues in the former at 4 weeks $(p<0.05)$ and 12 weeks $(p<0.001)$ postpartum, but not at 8 weeks postpartum. Suckling stimulated prolactin concentrations were also significantly higher ( $p<0.01$ ) in the LA group than in the SA group, and this difference was attributed to significantly higher $(p<0.01)$ prolactin concentrations at 12 weeks postpartum. Prolactin increments remained significantly different $(p<0.0001)$ between the two groups with the LA group having significantly higher prolactin increments at $4(p<0.05), 8(p<0.05)$ and 12 (p.0.01) weeks postpartum. Basal, suckling stimulated and the increment of prolactin significantly $(p<0.02$, $<0.01,<0.01$ respectively for linear trend) decreased with time postpartum in the SA group. In the LA group, basal concentrations and the increment of prolactin at 8 and 12 weeks postpartum were lower than at 4 weeks postpartum, but these differences were not statistically significant. In contrast, suckling stimulated prolactin concentrations remained similar from 4 to 12 weeks postpartum.

Neither the maternal BMI nor the infant weight expressed as a percentage of birth weight was significantly different between the two groups from 4 to 12 weeks postpartum (data not shown). Maternal BMI did not significantly change with time postpartum in either group, whereas the infant weight showed a significant $(p<0.001$ for lineartrend) increase in both groups as expected (data not shown).

Table 2. Infant feeding pattern in women who resumed regular menstruation before 24 weeks (SA group) and at or after 24 weeks (LA group) postpartum. Mean \pm SEM are shown

$\begin{array}{lllll}\text { Feeding variable } & \text { Group } & \begin{array}{l}4 \text { weeks } \\ \text { postpartum }\end{array} & \begin{array}{l}8 \text { weeks } \\ \text { postpartum }\end{array} & \begin{array}{l}12 \text { weeks } \\ \text { postpartum }\end{array} \\ \text { Total number of breastfeeds/24 h } & \text { SA group } & 12.00 \pm 0.94 & 9.80 \pm 0.62 & 9.67 \pm 0.67 \\ & \text { LA group } & 11.53 \pm 0.8 & 10.07 \pm 0.53 & 10.20 \pm 0.58 \\ \text { Number of daytime breastfeeds } & \text { SA group } & 8.92 \pm 0.83 & 7.20 \pm 0.59 & 7.71 \pm 0.68 \\ & \text { LA group } & 9.06 \pm 0.66 & 7.71 \pm 0.40 & 8.50 \pm 0.59 \\ \text { Number of night breastfeeds } & \text { SA group } & 3.43 \pm 0.37 & 2.57 \pm 0.34 & 2.07 \pm 0.32 \\ & \text { LA group } & 2.86 \pm 0.34 & 2.57 \pm 0.27 & 1.93 \pm 0.20 \\ \text { Number of other feeds/24 h } & \text { SA group } & 0 & 0.40 \pm 0.16 & 1.20 \pm 0.33 \\ & \text { LA group } & 0 & 0.33 \pm 0.16 & 0.40 \pm 0.16\end{array}$

Table 3. Plasma prolactin concentrations [geometric mean (95\% confidence limits)] in women who resumed regular menstruation before 24 weeks (SA group) and at or after 24 weeks (LA group) postpartum

$\begin{array}{lllll}\text { Prolactin concentration } & \text { Group } & \begin{array}{l}4 \text { weeks } \\ \text { postpartum }\end{array} & \begin{array}{l}8 \text { weeks } \\ \text { postpartum }\end{array} & \begin{array}{l}\text { I2 weeks } \\ \text { postpartum }\end{array} \\ \text { Basal } & \text { SA group } & 998(594-1675) & 753(435-1309) & 550(291-1035) \\ & \text { LA group } & 2133(1406-3243) & 1472(944-2296) & 1535(984-2388) \\ \text { Suckling stimulated } & \text { SA group } & 2167(1282-3672) & 1358(735-2511) & 1183(743-1884) \\ & \text { LA group } & 2540(1682-3828) & 2148(1213-3793) & 2636(1811-3846) \\ \text { Increment } & \text { SA group } & 783(334-1837) & 470(189-1169) & 466(232-933) \\ & \text { LA group } & 1318(887-1959) & 836(393-1789) & 908(551-1496)\end{array}$




\section{Discussion}

In this study, women who resumed menstruation before 24 weeks postpartum and those who resumed at or after 24 weeks had similar patterns of breastfeeding up to 8 weeks postpartum. At 4 weeks postpartum, infants in both groups were receiving only breast milk. Fruit juice and noncaloric liquids comprising less than $15 \%$ of all feeds were not regarded as "other feeds" in the present study, as exclusive breastfeeding and full breastfeeding, where up to 15 to $20 \%$ of other feeds are permitted, have shown similar endocrine characteristics $(3,17,18,19)$. From 8 weeks postpartum onwards, a few women in both groups introduced other feeds such as caloric liquids and formula feeds to their infants. However, this did not make a significant difference to the breastfeeding pattern at 8 weeks postpartum. But at 12 weeks postpartum women with short periods of lactational amenorrhoea gave a significantly higher number of other feeds compared to those with long periods of lactational amenorrhoea, resulting in a significant reduction in the percentage of breastfeeds in this group. As expected, the frequency of breastfeeds $/ 24 \mathrm{~h}$ significantly decreased and the frequency of other feeds significantly increased with time in both groups, the reduction being mainly due to reduction in the night breastfeeds. Daytime breastfeeds did not show any significant reduction from 4 weeks to 12 weeks postpartum in either group.

Despite the similarities in the breastfeeding pattern in both groups up to 8 weeks postpartum, those who had long periods of lactational amenorrhoea had significantly higher basal prolactin concentrations at 4 weeks postpartum, but they were not significantly different between the groups at 8 weeks postpartum. Women who had longer periods of lactational amenorrhoea were giving a significantly higher percentage of breastfeeds at 12 weeks postpartum. This was associated with significantly higher basal and suckling stimulated prolactin concentrations. The prolactin increment remained significantly higher in these women throughout the study period.

In a previous study (13) higher increments of prolactin, but similar basal prolactin were noted in women who resumed menstruation after 180 days postpartum when compared to those who resumed menstruation before 180 days. In a cross sectional study in a smaller number of women, even basal prolactin concentrations were higher in the long amenorrhoeics than in the short amenorrhoeics. The inability to observe such differences in the longitudinal study was attributed to prolactin estimation being performed on morning blood samples. Prolactin response to suckling is least evident in the morning (12). In the present study, blood sampling was in the afternoon for prolactin estimation. Although the basal prolactin concentrations were higher in the LA group throughout the study, this difference was statistically significant only at 4 and 12 weeks postpartum. The absence of a significant difference in basal prolactin at 8 weeks postpartum between the two groups in this study cannot therefore be attributed to the time of blood sampling. As in the previous study (13), significantly higher prolactin increments in the long amenorrhoeics were observed throughout the present study. This difference was evident at 4 weeks postpartum when both groups were fully breastfeeding as well as at 8 weeks and 12 weeks postpartum when the SA group was giving a higher number of other feeds (number of other feeds higher but not statistically significant at 8 weeks and significantly higher at 12 weeks postpartum). But Tay and co-workers (14) did not find any relationship between prolactin concentrations and the duration of lactational amenorrhoea when they studied 24-h prolactin secretory pattern during breastfeeding. In the present study, higher prolactin concentrations were observed in the LA group, even in the absence of any restrictions on infant feeding pattern. It is likely that the smaller number of subjects studied or the differences in the study design and statistical analyses used by Tay and co-workers (14) failed to bring out differences in prolactin concentrations between women who resumed menstruation early and late in the postpartum period.

Diaz and co-workers (13) also observed a higher mean number of nursing episodes $/ 24 \mathrm{~h}$ in the long amenorrhoeics than in the short amenorrhoeics, but analysis which took such factors into account still showed differences in prolactin concentrations between these two groups. In the present study there were no significant differences in the breastfeeding frequency between the two groups, though these were significantly higher in the LA group when expressed as a percentage of all feeds at 12 weeks postpartum. The frequency of other feeds and the percentage of feeds constituted by other feeds were significantly higher in the SA group at 12 weeks postpartum, confirming that early introduction of non-breast milk feeds is associated with shorter periods of lactational amenorrhea. In the study by Tay and co-workers (14), duration of lactational amenorrhoea strongly correlated to the time of introduction of supplementary feeds to the infant, but not to the frequency of suckling.

In the present study, there was a significant reduction in the breastfeeds (total/24 $\mathrm{h}$ and night) and a significant increase in the number of other feeds with time postpartum in both groups. But a significant reduction in prolactin concentrations (basal, suckling stimulated and the increment) with time postpartum was noted only in women with short periods of lactational amenorrhoea. Women in the LA group were able to maintain higher prolactin secretion in spite of a significant reduction in breastfeeding frequency with time postpartum, than those in the SA group. These findings suggest that there is an inherent difference in the prolactin response by the pituitary lactotrophe to the suckling stimulus between women who have short and long periods of lactational amenorrhoea, with the latter showing a greater response. However, we cannot exclude differences in the total duration of suckling/24 $\mathrm{h}$ or in the strength of the suckling stimulus between the two groups. The average duration of a suckling episode was obtained by recall, but this was not used for analysis, as almost all women reported this as 5 to $10 \mathrm{~min}$ utes. No attempt was made to quantify the strength of the suckling stimulus in view of the invasive nature of the methods available. 
The duration of lactational amenorrhoea correlates negatively with the maternal BMI and positively with parity in some studies, although disputed by others $(2,6,8$, $11,20)$. In the present study the LA group had a lower maternal BMI and the SA group had a higher number of para 3 women, but these differences were not statistically significant. The differences between the present and other studies may be partly due to the smaller number of subjects studied in the former. As in a previous study (11), we also failed to observe a significant difference in the sex of the infant in relation to the duration of lactational amenorrhoea. A strong positive correlation between the length of subsequent postpartum amenorrhoea has been previously reported (9). In the present study, a higher number of women in the SA group had a previous short lactational amenorrhoea, though the number of women with a previous long and short lactational amenorrhoea was similar (7 vs 8 ) in the LA group.

A variable which can be used during the early postpartum period to differentiate between women who will have short and long periods of lactational amenorrhoea would be of value in predicting those who will need contraception early. In view of the large individual variation in prolactin observed among lactating women prolactin measurement is unlikely to be useful in this respect.

\section{Acknowledgements}

I thank Prof. H R Seneviratne for giving access to postpartum women and Prof. E H Karunanayake for the laboratory facilities.

\section{References}

1. Howie PW, McNeilly AS, Houston MJ, Cook A, Boyle H. Fertility after childbirth: Infant feeding patterns, basal prolactin levels and postpartum ovulation. Clinical Endocrinology 1982; 17: 315-22.

2. Diaz S, Rodriguez G, Marshal G, del Pino G, Casado ME, Miranda $P$ et al. Breastfeeding pattern and the duration of lactational amenorrhoea in urban Chilean women. Contraception 1988; 38: 37-51.

3. Gray RH, Campbell OM, Apelo R, Eslami SS, Zacur H, Ramos RM et al. Risk of ovulation during lactation. Lancet 1990; 335: 25-9.

4. Howie PW, McNeilly AS, Houston MJ, Cook A, Boyle H. Effect of supplementary food on suckling patterns and ovarian activity during lactation. British Medical Journal 1981; 283: 757-9.

5. Whitehead RG, Rowland MGM, Hutton M, Prentice AM, Muller E, Paul A. Factors influencing lactation performance in rural Gambian mothers. Lancet 1978; 2: 178-81.

6. Prema K, Naidu AN, Neelakumari S, Ramalakshmi BA. Nutrition-fertility interaction in lactating women of low income groups. British Journal of Nutrition 1982; 45: 461-7.

7. Huffman SL, Ford K, Allen HA, Streble P. Nutrition and fertil- ity in Bangladesh: Breastfeeding and postpartum amenorrhoea. Population Studies 1987; 41: 447-62.

8. Popkin BM, Guilkey DK, Akin JS, Adair LS, Udry JR. Nutrition, lactation and birth spacing in Filipino women. Demography 1993; 30: 333-52.

9. Ford K. Correlation between subsequent lengths of postpartum amenorrhoea in a prospective study of breastfeeding women in rural Bangladesh. Journal of Biosocial Science 1992; 24: 89 95.

10. World Health Organisation Task Force on Methods of Natural Regulation of Fertility. The World Health Organization multinational study of breastfeeding and lactational amenorrhoea $I$. Description of the infant feeding patterns and of the return of menses. Fertility and Sterility 1998 a; 70: 448-60.

11. World Health Organisation Task Force on Methods of Natural Regulation of Fertility. The World Health Organisation multinational study of breastfeeding and lactational amenorrhoea II. Factors associated with the length of amenorrhoea. Fertility and Sterility 1998 b; 70: 461-71.

12. Diaz S, Serono-Ferre M, Cardenas H, Schiappacasse V, Brandeis A, Croxatto HB. Circadian variation of basal plasma prolactin response to suckling and length of amenorrhoea in nursing women. Journal of Clinical Endocrinology and Metabolism 1989; 68: 946-55.

13. Diaz S, Cardenas H, Brandeis A, Miranda P, Schiappacasse V, Salvatierra AM et al. Early differences in the endocrine profile of long and short lactational amenorrhoea. Journal of Clinical Endocrinology and Metabolism 1991; 72: 196-201.

14. Tay CCK, Glasier AF, McNeilly AS. Twenty-four hour patterns of prolactin secretion during lactation and the relationship to suckling and the resumption of fertility in breastfeeding women. Human Reproduction 1996; 11: 950-5.

15. Tennekoon KH, Karunanayake EH, Seneviratne HR. Effect of skim milk supplementation of the maternal diet on lactational amenorrhoea, maternal prolactin, and lactational behaviour. American Journal of Clinical Nutrition 1996; 64: 283-90.

16. Sufi SB, Donaldson A, Jeffcoate SL. World Health Organization Special Programme on Research, Development and Research training in Human Reproduction, Programme for the Provision of Matched Reagents for the Radioimmunoassay of Hormones in Reproductive Physiology, Method Manual, 1991.

17. Kennedy KI, Rivera R, McNeilly AS. Consensus statement on the use of breastfeeding as a family planning method. Contraception 1989; 39: 447-96.

18. Labbok $M$, Krasovec $K$. Towards consistency in breastfeeding definitions. Studies in Family Planning 1990; 21: 226-30.

19. Campbell OMR, Gray RH. Characteristics and determinants of postpartum ovarian function in women in the United States. American Joumal of Obstetrics and Gynaecology 1993; 169: 55-60.

20. Kurz KM, Habicht J-P, Rasmussen KW, Schwager SJ. Effect of maternal nutritional status and maternal energy supplementation on length of postpartum amenorrhoea among Gautemalan women. American Journal of Clinical Nutrition 1993; 58: 63640. 